University of Wollongong

Research Online

Australian Institute for Innovative Materials -

Papers

Australian Institute for Innovative Materials

$1-1-2013$

\title{
Surface properties and interaction forces of biopolymer-doped conductive polypyrrole surfaces by atomic force microscopy
}

Jani M. Pelto

VTT Technical Research Centre of Finland, jani@uow.edu.au

Suvi P. Haimi

University of Twente

Aliisa S. Siljander

VTT Technical Research Centre of Finland

Susanna S. Miettinen

VTT Technical Research Centre of Finland

Kirsi M. Tappura

VTT Technical Research Centre of Finland

See next page for additional authors

Follow this and additional works at: https://ro.uow.edu.au/aiimpapers

Part of the Engineering Commons, and the Physical Sciences and Mathematics Commons

Research Online is the open access institutional repository for the University of Wollongong. For further information contact the UOW Library: research-pubs@uow.edu.au 


\title{
Surface properties and interaction forces of biopolymer-doped conductive polypyrrole surfaces by atomic force microscopy
}

\begin{abstract}
Surface properties and electrical charges are critical factors elucidating cell interactions on biomaterial surfaces. The surface potential distribution and the nanoscopic and microscopic surface elasticity of organic polypyrrole-hyaluronic acid (PPy-HA) were studied by atomic force microscopy (AFM) in a fluid environment in order to explain the observed enhancement in the attachment of human adipose stem cells on positively charged PPy-HA films. The electrostatic force between the AFM tip and a charged PPyHA surface, the tip-sample adhesion force, and elastic moduli were estimated from the AFM force curves, and the data were fitted to electrostatic double-layer and elastic contact models. The surface potential of the charged and dried PPy-HA films was assessed with Kelvin probe force microscopy (KPFM), and the KPFM data were correlated to the fluid AFM data. The surface charge distribution and elasticity were both found to correlate well with the nodular morphology of PPy-HA and to be sensitive to the electrochemical charging conditions. Furthermore, a significant change in the adhesion was detected when the surface was electrochemically charged positive. The results highlight the potential of positively charged PPy-HA as a coating material to enhance the stem cell response in tissue-engineering scaffolds. 2013 American Chemical Society.
\end{abstract}

\section{Keywords}

surfaces, polypyrrole, conductive, doped, biopolymer, forces, atomic, force, surface, properties, interaction, microscopy

Disciplines

Engineering | Physical Sciences and Mathematics

\section{Publication Details}

Pelto, J. M., Haimi, S. P., Siljander, A. S., Miettinen, S. S., Tappura, K. M., Higgins, M. J. \& Wallace, G. G. (2013). Surface properties and interaction forces of biopolymer-doped conductive polypyrrole surfaces by atomic force microscopy. Langmuir, 29 (20), 6099-6108.

\section{Authors}

Jani M. Pelto, Suvi P. Haimi, Aliisa S. Siljander, Susanna S. Miettinen, Kirsi M. Tappura, Michael J. Higgins, and Gordon G. Wallace 


\section{Surface properties and interaction forces of}

\section{biopolymer doped conductive polypyrrole surfaces}

\section{by atomic force microscopy}

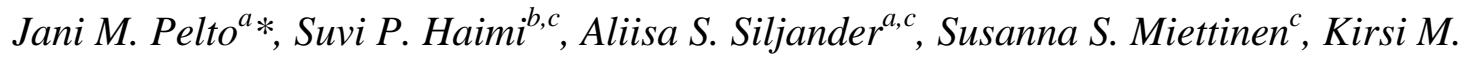
Tappura $^{a}$, Michael J. Higgins ${ }^{d}$, Gordon G. Wallace ${ }^{d}$

${ }^{\mathrm{a}}$ VTT Technical Research Centre of Finland, Sinitaival 6, P.O. Box 1300, 33101 Tampere, Finland

${ }^{\mathrm{b}}$ Department of Biomaterials Science and Technology, University of Twente, Enschede, P.O. Box 217, 7500 AE Enschede, The Netherlands

${ }^{\mathrm{c}}$ Adult Stem Cells, Institute of Biomedical Technology, Biokatu 8, FI-33014 University of Tampere, Finland

${ }^{\mathrm{d}}$ ARC Centre of Excellence for Electromaterials Science (ACES), Intelligent Polymer Research Institute (IPRI), Australian Institute for Innovative Materials (AIIM) Facility, Innovation Campus, University of Wollongong, NSW, 2522, Australia. 


\section{KEYWORDS}

atomic force microscopy, adipose stem cell, polypyrrole, hyaluronic acid

\section{ABSTRACT}

Surface properties and electrical charges are critical factors elucidating cell interactions on biomaterial surfaces. The surface potential distribution as well as the nanoscopic and microscopic surface elasticity of organic Polypyrrole-Hyaluronic acid (PPy-HA) were studied by atomic force microscopy (AFM) in fluid environment in order to explain the observed enhancement in the attachment of human adipose stem cells on positively charged PPy-HA films. The electrostatic force between the AFM tip and a charged PPy-HA surface, the tipsample adhesion force and elastic moduli were estimated from the AFM force curves and the data fitted to electrostatic double layer and elastic contact models. Surface potential of the charged and dried PPy-HA films was assessed with Kelvin probe force microscopy (KPFM) and the KPFM data correlated to the fluid AFM data. The surface charge distribution and elasticity were both found to correlate well with the nodular morphology of PPy-HA and to be sensitive to the electrochemical charging conditions. Furthermore, a significant change in the adhesion was detected when the surface was electrochemically charged positive. The results highlight the potential of positively charged PPy-HA as a coating material to enhance stem cell response in tissue engineering scaffolds. 


\section{Introduction}

Polypyrrole (PPy) is a prototype of electroactive organic p-type semiconductor that is widely applied as a surface coating on a range of metallic conductors and insulative biomaterials such as tissue engineering scaffolds. ${ }^{1}$ The electroactivity of PPy stems from the property of the neutral and electrically insulating $\mathrm{PPy}^{0}$ chains becoming positively charged (polaronic $\mathrm{PPy}+$ ) and electronically conductive when oxidized in electrolytes. The charge of the PPy+ backbone is always compensated by negatively charged species, called dopants, or ions from the surrounding electrolyte. Any excess surface charge of electrochemically charged polypyrrole is not only dependent on the bulk doping charge of the polymer but also on the surface chemistry in the electrolyte. Factors such as the mobility of the dopants and properties of the electrolyte solution, e.g. salt concentration and $\mathrm{pH}$, are involved in the surface chemistry. ${ }^{2}$

We have previously reported that PPy doped with Hyaluronic Acid (HA) show potential as a substrate material for supporting the growth of human adipose stem cells (hASC). ${ }^{3}$ HA is an extracellular matrix polysaccharide that has widely been studied as a biocompatible polymeric dopant of polypyrrole. It is an extremely hydrophilic anionic polymer that renders the polymer (i.e. PPy-HA) very hydrophilic, and under certain synthetic conditions, a soft polymer with the Young's modulus in the range of megapascals can be produced. According to its charging state, PPy-HA may reversibly absorb salt and water from the surrounding electrolyte, a process that contributes to the elasticity of the polymer surface. ${ }^{4}$ Serra Moreno et al. ${ }^{5}$ have studied the effects of synthesis parameters of polysaccharide doped PPy, including the effect of their surface roughness, on osteoblast adhesion and proliferation. Their findings suggest that smooth surfaces allow the best adhesion of osteoblasts on electropolymerized films. A similar PPy-HA coating has been recently designed to inhibit unwanted adhesion of astrocytes and fibroblasts onto neural 
probes. ${ }^{6}$ Cell interactions with PPy-HA coated surfaces are likely to depend not only on the specific chemistry of the dopant type but also on other physical surfaces properties (modulus, surface potential, morphology) which are influenced by the presence of the dopant and parameters (e.g. polymerization charge of polymer growth) used to synthesize the polymer.,

However, there are still gaps in the fundamental understanding of cell-surface interactions at the single molecular (atomic and nanoscopic) level and micron scales related to processes involving initial cell attachment, spreading and adhesion. Therefore, the role of the interface and its various surface properties, including nanoscale and microscale surface roughness, elasticity and electrical charges, need further elucidation in order to fully understand cell interactions on biomaterials. This is particularly the case given the ongoing emergence of new and diverse biomaterials, including electroactive polymers that are continually under development due to their easy modification, functionalization and doping with biological constituents (e.g. glycosaminoglycans, drugs, proteins). Whilst some studies on electroactive polymers have examined the effect of their physical surface properties on cell interactions ${ }^{1,3,5,6}$, such as proliferation, adhesion and differentiation, few studies in this context have been undertaken using approaches that quantify and correlate variations in nanoscale surface properties. This said, many studies using Atomic Force Microscopy (AFM) have shown that electroactive polymers display characteristic nanoscale lateral variations, or effective phase separations, of the modulus, surface potential, conductivity and interaction forces, that occur across the polymer surface and are related to its nanoscale morphology. ${ }^{7}$

Of particular interest is how these nanoscale properties manifest under natural physiological conditions, in particular, variations in surface charge arising spontaneously on ionized chemical groups and electrical double layers at surfaces immersed in physiological fluid. Electrical double 
layer forces, strong van der Waals interactions and steric forces are present in colloidal aqueous systems, including biological interfaces. Hence, the physical interactions between the continuum of biomaterial surfaces, plasma proteins and surface receptors of living cells are clearly attributable to their electrostatic charges and/or hydrated polymers on their surfaces. ${ }^{9}$ Lubarsky et al. ${ }^{10}$ have shown that electrical double layer forces drive human serum albumin adsorption more efficiently onto non-oxidized and less negatively charged tissue culture polystyrene plates in phosphate buffered saline (PBS). According to a recent review (see Dubiel, Martin, and Vermette ${ }^{9}$ ), the importance of the electrostatic double layer forces on adhesion proteins and cell adhesion has been well-stated in recent studies.

Surface charges measured with different analytical techniques are quite different. Atomic force microscopy (AFM) measurements in fluid yield "effective" nanoscopic information on the surface charge/potential based on Gouy-Chapman-Stern (GCS) treatment of the diffuse electrical double layer, whereas electrokinetic measurements yield information of the averaged surface zeta-potential, which by definition are different quantities. Both of these parameters have been used to describe the attachment of charged biopolymers and cells on biomaterials, but neither can be directly deduced from the electrochemical (bulk or surface) charge injected into the biomaterial and/or the properties of the physiological electrolyte. As there is no universally applicable theory available linking the electrochemical charge and the effective surface charge and -potential, they must be determined using some probing technique, such as AFM. ${ }^{11}$ Hence, the role of the surface charge in adhesion of biopolymers in biological environments, in particular for electroactive polymers on the nanoscale, has remained difficult to directly quantify and their effects on biological interactions unclear. 
The significance of gaining an improved understanding of nanoscale surface forces is evident from the mechanisms of cell attachment and spreading that require the initial adsorption of ECM proteins followed by the application of piconewton, or even nanonewton, forces to the substrate via focal adhesion complexes, all of which are also influenced by the elastic response of the

substrate. ${ }^{12-13}$ Emerging evidence suggests that the mechanochemical response originating from the cell-substrate interactions is specific to cell phenotype, the understanding of which has already been utilized to great effect in the differentiation and sorting of cell types ${ }^{12}$ and could also be applied to electroactive polymers through modulation of the redox surface properties.

In this study, we have utilized Kelvin force probe microscopy (KFPM) and AFM to elucidate nanoscopic lateral variations in the roughness, elasticity and surface charge across the surface of PPy-HA substrates as a function of the redox state of the polymer. In particular, we attempt to relate these variations in surface properties, in addition to the surfaces forces operating in fluid, to observed differences in human adipose stem cell (hASC) adhesion and spreading on thin potentiostatically electropolymerized and charged PPy-HA surfaces.

\section{Materials and Methods}

\subsection{Polypyrrole-hyaluronic acid synthesis}

Polypyrrole samples were electropolymerized onto indium tin oxide (ITO) substrates (surface resistance $40 \Omega$ /sq, Sigma Aldrich, St. Louis, USA) using potentiostat (Parstat 261A, EG\&G, Oak Ridge, USA). Another set of sample for the electrical characterization was grown on AuMylar (RF-sputtered $50 \mathrm{~nm} \mathrm{Au,} \mathrm{VTT,} \mathrm{Finland)} \mathrm{films.} \mathrm{For} \mathrm{the} \mathrm{electropolymerization,} \mathrm{0.2} \mathrm{M}$ distilled pyrrole (Sigma) was dissolved in $1 \mathrm{mg} / \mathrm{mL}$ solution of HA extracted from human umbilical cord (Sigma). Constant potential $+1.0 \mathrm{~V}$ vs. $\mathrm{Ag} / \mathrm{AgCl}$ was applied to the working electrode substrate. Platinum counter electrode was used. The polymerization was continued 
until a polymerization charge $0.5 \mathrm{C} / \mathrm{cm}^{2}$ had been consumed. At this point the PPy-HA film thicknesses were 600-800 nm, as confirmed by AFM.

After the synthesis, the PPy-HA samples were rinsed with deionized water and dried in air. Samples grown on ITO substrates were gamma-sterilized (radiation dose $20 \mathrm{kGy}$ ) before cell seeding or before $2 \mathrm{~d}$ pre-incubation period in maintenance medium consisting of DMEM/F-12 1:1 (Invitrogen, Paisley, UK), 10\% FBS (Invitrogen), 1\% L-glutamine (GlutaMAX I; Invitrogen) and 1\% penstrep (100 U/mL penicillin, $0.1 \mathrm{mg} / \mathrm{mL}$ streptomycin; Invitrogen). Corresponding PPy-HA films grown on Au-Mylar were used in the electrical characterization.

\subsection{Electrochemical charging}

We examined the effect of the charged state of PPy-HA on hASCs adhesion. For charged samples, the PPy-HA films were electrochemically charged by applying $+200 \mathrm{mV}$ vs. Ag/AgCl reference directly before seeding hASCs and during the cell culture period (Table 1). For uncharged samples (defined as samples where $+200 \mathrm{mV}$ was not applied), the as-synthesized films were connected to a reference $+0 \mathrm{mV}$ electrode. Therefore, both groups of samples were to set to a potential during the cell culture. For the AFM experiments, the charged and uncharged samples were identically prepared, with the charged samples being subject to the application of $+200 \mathrm{mV}$ vs. Ag/AgCl (0.005M NaCl) in the AFM electrochemical cell shortly before the AFM scan. The sample potential was controlled using an EDAQ EA161 potentiostat and platinum wire counter electrode immersed into the AFM electrochemical cell. During the AFM scan the sample potential was not controlled.

\subsection{Cell culture}

\subsubsection{Human adipose stem cells}


Human ASCs were isolated from the adipose tissue samples of subcutis collected from surgical procedures from two females (ages 45 and 50), kindly provided by the Department of Plastic Surgery, Tampere University Hospital. Isolation of hASCs from the tissue samples was conducted in accordance with the Ethics Committee of Pirkanmaa Hospital District, Tampere, Finland (R03058). The adipose tissue was digested with collagenase Type I $(1.5 \mathrm{mg} / \mathrm{mL}$; Invitrogen). Human ASCs were expanded in T-75 polystyrene flasks (Nunc, Roskilde, Denmark) in maintenance medium. Cells of passages 5-7 were used in the experiments. Cells were seeded to each PPy-HA sample in a medium volume of $1 \mathrm{~mL}, 25,000$ cells per sample and cultured up to $3 \mathrm{~h}$.

The hASCs of passage 2-3 were harvested and analyzed by flow cytometry (FACSAria; BD Biosciences, Erembodegem, Belgium). Monoclonal antibodies against CD9-PE, CD10-PECy7, CD13-PE, CD29-APC, CD49d-PE, CD90-APC, CD106-PE-Cy5, CD146-PE and CD166-PE (BD Biosciences); CD45-FITC (Miltenyi Biotech, Bergisch Gladbach, Germany); CD31-FITC, CD34-APC, CD44-FITC and HLA-ABC-PE (Immunotools GmbH Friesoythe, Germany); and CD105-PE (R\&D Systems Inc, MN, USA) were used. Analysis was performed on 10,000 cells per sample and unstained cell samples were used to compensate for the background autofluorescence levels. The flow cytometric analysis on hASCs demonstrated expression for markers substantiating mesenchymal origin of cells and showing low or lack of expression of markers suggesting hematopoietic and angiogenic origin of cells. The characterization data comply with existing results on hASCs. ${ }^{14-16}$

\subsubsection{Cell attachment and viability}

Cell attachment and viability were studied using Live/Dead staining (Invitrogen) according to the manufacturer's protocol at $3 \mathrm{~h}$ time point. The viable cells (green fluorescence) and dead cells 
(red fluorescence) were examined using a fluorescence microscope. For AFM visualization, similarly treated samples as for Live/Dead staining were fixed before imaging. After the $3 \mathrm{~h}$ culture period, cell-seeded samples were washed with DPBS and fixed with 5\% glutaraldehydefixative in $0.1 \mathrm{M}$ phosphate buffer ( $\mathrm{pH} 7.4$ ) for $48 \mathrm{~h}$. 5\% glutaraldehyde cell fixation is a standard cell fixation method which we have used in our previous studies with human adipose stem cells for morphology imaging. ${ }^{17,18}$ Fixative was replaced by Dulbecco’s phosphate buffered saline (DPBS), where samples were stored until imaging. Three to five representative optical images of the cells, selected from different areas of each PPyHA sample, were analyzed to measure the surface area of the individual cells $(n=26)$ using i-Solution Lite image acquisition and measuring software (IMT i-Solutions Inc.).

\subsection{Characterization of the polypyrrole films}

\subsubsection{Kelvin force probe microscopy}

For the KFPM analysis the dried samples were first immersed in dilute $0.005 \mathrm{M} \mathrm{NaCl}$ electrolyte. Subsequently, the samples were either charged (+200 mV vs. Ag/AgCl) or shortcut

to the Ag-pseudo-reference electrode (0 mV vs. Ag). The samples were carefully rinsed to remove any salt solution and left to dry under ambient conditions. The surface charge distribution of the PPy-HA samples in air was measured using an Asylum MPF-3D AFM in the standard Kelvin force probe mode. Olympus OMCL-AC240TM probes with a platinum coating, cantilever spring constant of $\sim 2 \mathrm{~N} / \mathrm{m}$ and resonance frequency of $70 \mathrm{kHz}$ were used for imaging at a scan rate of $0.5 \mathrm{~Hz}$. The topography of the sample area was acquired in AC-mode whilst simultaneously recording the surface potential of the polymer films. Relative differences in the surface potential across the films were obtained.

\subsubsection{AFM force spectroscopy}


AFM force-distance curves were acquired using an Asylum MPF-3D AFM in the force volume mode. The measurements were done in dilute $0.005 \mathrm{M} \mathrm{NaCl}$ electrolyte in an open measurement cell at room temperature. Silver wire was used as a pseudo-reference electrode in those experiments with a biased AFM tip.

Characterization of the surface charge was done using biased $(-200 \mathrm{mV})$ or neutral $(0 \mathrm{mV})$ gold-coated silicon nitride tips OBL (Olympus Corp, Tokyo, Japan). The spring constant of the OBL cantilever was determined to be $0.020 \mathrm{~N} / \mathrm{m}$ by the Sader method ${ }^{19}$ and the cantilever sensitivity in the electrolyte was measured from the slope of the recorded force curves. The low concentration of salt $(0.005 \mathrm{M})$ was applied in order to be able to project the electrostatic interactions to long distances where they can be distinguished from hydration and steric forces. Negative or zero bias voltage kept the tip-sample interaction in the repulsive regime indicating negative surface charge of PPy-HA. The negative surface charge was confirmed by initial testing with both positive and negative bias on the tip, of which only the negative bias resulted in repulsive interaction. A maximum applied force of $0.7 \mathrm{nN}$ was kept constant for the individual curves, which were obtained at a scan rate of $300 \mathrm{~nm} / \mathrm{s}$. 32x32 force curve maps were collected for both the neutral $(0 \mathrm{mV})$ and negatively biased $(-200 \mathrm{mV})$ tip. To qualitatively elucidate the effect of electrostatic double layer forces on the tip-sample interaction under physiological conditions, the force curve data was fitted to two simple DLVO models. ${ }^{20,21}$ The DLVO fitting gave estimates on the range of the repulsion forces for comparison with the theoretically calculated double layer. In addition, the polarity and relative magnitude of the excess surface charge density (surface potential) could be estimated.

The surface elasticity was measured using platinum-iridium coated pyramidal silicon tips ANSCM-PC (Applied Nanostructures Inc., Santa Clara, CA, USA). A maximum applied force of 
1-1.5 $\mathrm{nN}$ was kept constant for the individual curves, which were obtained at a scan rate of 300 $\mathrm{nm} / \mathrm{s}$. 64x64force curve maps were collected with a neutral $(0 \mathrm{mV})$ tip. The spring constant of the ANSCM-PC cantilever was determined to be $0.034 \mathrm{~N} / \mathrm{m}$ by the Sader method ${ }^{19}$ and the cantilever sensitivity in the electrolyte was measured from the slope of the recorded force curves. The force curves were converted to force versus indentation curves and fitted with the Hertz Model to quantify the Young's modulus. The analysis procedure was carried out using the Asylum AFM Software (Igor Pro, Wavemetrics) and according to previously described methods. $^{7}$

\subsection{Statistical analyses}

Statistical analyses were performed using Origin Pro (Originlab Corporation, Northampton,

MA, USA), version 8. The statistical significance of cell spreading data was assessed using Student's t-test and ANOVA at a significance level of 0.01. Levene’s Test for equal variance and Bonferroni’s post hoc correction and test for equal means were used in the ANOVA.

The significance of possible variation in the surface potential derived from the AFM force spectroscopy data was assessed by Student's t-test at a significance level of 0.05.

\section{Results and Discussion}

\subsection{Cell attachment and viability}

Spreading of hASCs on non-preincubated (1A and B), pre-incubated (1C and D) and either charged (1B and $\mathrm{D})$ or uncharged (1A and C) PPy-HA surfaces are presented in the representative optical images (Fig.1A-D, left panel). The majority of hASCs were uniformly adhered at the 3h time point on charged samples surfaces. However, hASCs seeded on noncharged PPy-HA surfaces were unevenly spread and many cells were incompletely attached. 
Analysing the average cell areas showed that both the pre-incubation and charging influenced the cell spreading significantly (Fig.2): Student's t-test statistics for equal means between preincubated groups (+/pre-inc) vs. (0/pre-inc) gave $p<.001$, between non pre-incubated groups $(+)$ vs. (0) $p<.001$, and between groups (+/pre-inc) and (0) $p<.001$. The effect of the pre-incubation and the charging compared in the groups (0/pre-inc) vs. (+) were not statistically significant $(p=0.28)$. Qualitatively, the charged (+200 mV) PPyHA samples supported hASC attachment and spreading notably more than the non-charged $(0 \mathrm{mV})$ samples.

Levene's Test indicated significantly different variances $F(3,135)=28.8, p<.001$. According to one-way ANOVA the average cell areas were significantly different between the groups $F(3,135)=55.1, p<.001$. Bonferroni test indicated that the difference between the groups (0/preinc) and (+) was not significant at level of 0.01 , which was consistent with the result of the pairwise t-test.

In summary, the combined effect of pre-incubation and charging resulted in $215 \%$ increase of the hASC surface area compared to non-pre-incubated and non-charged control. Viability of the hASCs was not significantly affected by charging or the pre-incubation of the PPy surfaces, as indicated by live/dead images (Fig 1A-D, right panel).

Therefore, a main finding from these observations is that the charged surfaces, even those not exposed to serum proteins for longer incubation times in media, promoted cell adhesion to a greater extent than uncharged samples treated under the same conditions. This suggest that the application of a positive bias of $+200 \mathrm{mV}$ was beneficial in promoting cell adhesion, which may have occurred by either increased adsorption of serum proteins (e.g. fibronectin, vitronectin) during pre-incubation, and/or immediately upon cell seeding and settling over 3h, or through direct electrostatic interactions with the cell membrane. ${ }^{22}$ The effect of pre-incubation on cell 
attachment was not as clear as the effect of charging, although pre-incubation seemed also facilitate the cell adhesion to the polymer.

\subsection{Characterization of the surface charge}

\subsubsection{Kelvin Force Probe Microscopy}

AFM topography images (left) and their corresponding surface potential maps (right) of uncharged $(+0 \mathrm{mV})$ (Fig. 3A and B) and charged (+200 mV) (Fig. 3C and D) PPy-HA obtained from KFPM are presented in the figure 3. The root mean square (RMS) (average) roughness values of the uncharged and charged were $8.9 \mathrm{~nm}$ and $9.4 \mathrm{~nm}$, respectively, indicating that the surface roughness was not significantly affected by the electrochemical charging. These roughness values, along with the geometric area of the images, also equate to comparable surface area values of $4.52 \mu \mathrm{m}^{2}$ (uncharged) and $4.57 \mu \mathrm{m}^{2}$ (charged). The surface potential images in figures 3B and 3D display relative (not absolute) changes in the surface potential across the uncharged and charged films, with average variations of $4.38 \mathrm{mV}$ RMS and $2.91 \mathrm{mV}$ RMS, respectively. As evident in the surface potential images, the uncharged $(+0 \mathrm{mV})$ showed phase separation in surface potential where more negative potentials (darker regions in Fig. 3B) correlated with the nodule structures, while areas of more positive surface potential correlated with the peripheries of the nodules (brighter regions in Fig. 3B). In contrast, the surface potential of the charged films showed a more uniform distribution of the surface potential with no clear correlation with the topography (Fig. 3D). In some cases, it did appear that the larger nodules showed a more positive potential than the surrounding areas. These observations are confirmed also in previous studies using $\mathrm{KPFM}^{23}$, conductive- $\mathrm{AFM}^{24}$ and recently by $\mathrm{AFM}$ phase

imaging. ${ }^{7,25}$ Previous observations in the surface potential, conduction current and AFM phase 
signals showing that the application of a positive electrochemical potential largely removes this phase separation, is in accordance with the observations of this study. Many of these studies, as well as ours here, support the presence of more crystalline, heavily doped and electrically conductive parts of the PPy-HA in the nodules. In this study, we also applied a positive bias to the PPy-HA films with a moderate oxidizing potential (+200 mV vs. Ag) in the presence of a dilute saline electrolyte to avoid overoxidation of the polaronic PPy+ chain and accumulation of excess salt on the PPy-HA surface upon drying. It is likely that charge compensation by sodium and chloride ions in the dilute electrolyte would have occurred immediately and therefore the extent and role of the negatively charged HA molecules remained unclear in the PPy. We have learned from our previous X-ray fluorescence elemental analysis (unpublished data) that significant amount of chloride ions remains in the PPyHA films after rinsing with water. However, the PPyHA surface remained extremely hydrophilic implying that the surface exposed to the electrolyte contains significant amounts of HA fragments and/or -polymers. Nevertheless, it is possible that that ion exchange between the chloride ions and acid groups of the HA were at least partly responsible for the structural and electrical reorganization observed in this study and the preceding studies. ${ }^{7,22-25}$

\subsubsection{AFM Force Mapping}

Force maps for the uncharged and charged samples with no potential bias applied to the tip and obtained in $5 \mathrm{mM} \mathrm{NaCl}$ are shown in Figures 4 and 5, respectively. For the uncharged sample, the topography image gave an RMS (average) surface roughness of $23.7 \mathrm{~nm}$ (surface area, $1.23 \mu \mathrm{m}^{2}$ ) (Fig. 4A). As each pixel in the topography image represents a single force curve, we were able to correlate the latter with specific $x-y$ positions on the film. The inset in figure 4B shows two force curves taken on a nodule structure (position 1) and the periphery of the nodules 
(position 2) from the marked region (red square) in the topography image. Neither of the curves exhibited adhesion but the slope of their contact regions differed (Figure 4B). The slope of the contact region in the force curves for the nodule structure (solid line) was steeper compared to the nodule periphery (dashed line), indicating that former had a higher stiffness. Variation in both the adhesion and stiffness properties, extracted from the force curves, could be further assessed as a function of the morphology by plotting these two parameters as function of the $x-y$ coordinates to display adhesion (Figure 4C and D) and Young's modulus (Figure 4E and F) maps. Figure 4C of the adhesion map, which is also overlaid on a 3-dimensional topography image in Figure 4D, showed low adhesion (RMS average, $45 \mathrm{pN}$ ) between the tip and polymer and no correlation with the surface topography. A higher modulus (lighter areas) was observed at the nodule structures compared to their peripheries (Figure 4E and $\mathrm{F}$ ), indicating a phase separation in the stiffness of the film. The RMS (average) values of the Young's modulus estimated from the AFM analysis gave values of $2.3 \mathrm{MPa}$, which is very low compared to previously measured Young's modulus values for electroactive polymers. ${ }^{7}$ In comparison, the charged sample showed the same morphology and similar RMS (average) roughness of $29.2 \mathrm{~nm}$ (surface area, $1.28 \mu^{2}$ ) (Fig. 5A), indicating that the charging of the film did not have a significant effect in the surface topography. Individual force curves on the nodules (position 1, inset from the marked region in Fig. 5A) showed no adhesion (Fig. 5B, solid curve) but in this case adhesion was observed at the nodule periphery. This was clearly observed in the adhesion maps (Fig. 5C) and adhesion overlay with the topography (Fig. 5D), which showed significantly greater adhesion (RMS $634 \mathrm{pN}$ ) that strongly correlated with regions of only the nodule peripheries. The charged surface had a slightly higher Young's modulus (RMS average, 5.5 MPa) than the uncharged surface though both of the surfaces showed that the nodule regions 
(RMS average, 5.4 MPa) were stiffer than their peripheries (RMS average 2.3 MPa), see figures $5 \mathrm{E}$ and $\mathrm{F}$ and figures $4 \mathrm{E}$ and $\mathrm{F}$.

Although the increase in the modulus for the charged samples was not significant, the oxidation-reduction cycling of conducting polymers has previously been shown to induce significant changes in the stiffness of PPy films. ${ }^{3,4,7,26}$ It has been well stated in the literature that both the osmotic pressure mediated by hydration water and ion concentration gradients of the electrolyte salt and/or inherent stiffness change of the polypyrrole chains in response to doping are relevant factors explaining the observed changes in the elasticity in PPy doped with sulfonic acids. ${ }^{26}$ For PPy-HA in aqueous $\mathrm{NaCl}$ electrolyte, the exchange of both $\mathrm{Na}^{+}$and $\mathrm{Cl}^{-}$-ions (and their hydration water), driven by the Donnan potentials of the ions is likely to occur. ${ }^{26}$ However, we did not observe significant change in the Young's modulus attributable to excessive concentration of $\mathrm{Na}^{+}$(or $\mathrm{Cl}^{-}$)ions within the PPy-HA upon charging. Interestingly, the Young's modulus values measured for PPy-HA in this study are two orders of magnitude lower than previously reported values. For example, the incorporation of HA into PPy was qualitatively shown to result in rougher and more brittle films when compared to PPy-PSS films ${ }^{1}$, while Gelmi et al. ${ }^{7}$ obtained values of $600-700$ MPa for in phosphate buffer saline using similar AFM approaches. The large variation in the reported Young's moduli is attributable to the different polymerization methods and -reagents, especially in the origin and molecular weight of the HA. The thickness of the PPy-HA films of this study was significantly greater than in the previous studies. ${ }^{15,7,25}$ The typical Young's moduli of conducting polymers, including PPy and commonly applied poly(3,4-ethylenedioxythiophene) (PEDOT), on the order of several hundred MPa to a few GPa remains one caveat in their use as biomaterials, as there is often a modulus mismatch between the polymer and much softer target tissue (e.g. nerve, muscle). Therefore, our 
particularly low modulus values (2-5 MPa) are representative of more mechanically compatible conducting polymers with the values even lower than PPy doped with poly(methoxy aniline sulfonate) (PMAS) (30 MPa) whereby the PMAS introduces hydrogel-like properties and a high water content (>90\%). An important conclusion that could be drawn from the above force maps is that charging of the films did not have a significant effect on the topography and Young's Modulus but clearly increased the adhesion, specifically at the nodules, between the AFM tip and polymer. By excluding the topography and Young's modulus, we suggest that the interactions (e.g. electrostatic forces) and surface properties related to increased adhesion on the charged films are likely to be responsible for increased cell spreading and adhesion on these films, as observed in Table 1 and Figure 1.

Compared to the previous force maps, an applied bias to the tip enabled further investigation of the interaction forces, particularly electrostatic and adhesion, by providing a known surface potential at the AFM tip that could be controlled externally. Figure 6 and 7 present a total of 20 representative force curves for the uncharged (0 mV) and charged (+200 mV) PPy-HA surfaces, respectively, obtained from force maps taken in $5 \mathrm{mM} \mathrm{NaCl}$ with a bias of $-200 \mathrm{mV}$ applied to the AFM tip. Force curves were selected either from the nodules (Figure 6A and 7A) or from the peripheries of the nodules (Figure 6B and 7B). For the uncharged surfaces, a repulsive interaction upon approach and no adhesion were observed on the nodules, whereas in contrast, an increased 'snap-in' or small-range attractive force upon approach and adhesion of $\sim 350 \mathrm{pN}$ RMS were observed in the peripheries (Fig. 6A and B). In particular, we show below that the observed repulsive interactions for both the nodules and their peripheries are due to electrostatic forces and suggest that the small range attractive forces occurring only on the peripheries contributes to the observed adhesion (Fig. 6B). When the film was charged (+200 mV), small- 
range attractive forces upon approach and increased adhesion appeared on the nodules and remained on the nodule peripheries (Fig. 7). The appearance of small-range attractive forces on the nodule areas again correlates with adhesion at these locations. Furthermore, the effect of charging on the surface potential in the KFPM images and above surface forces is clearly more pronounced at the nodule regions. Occasionally multiple, small-range attractive forces were observed on the charged films, indicating that some of these interactions were attributable to the tip penetrating the sample surface. Indeed, the observed low elastic modulus of these films makes them susceptible to penetration by the AFM tip, which may contribute to the adhesion. The presence of these multiple 'snap-ins' however makes the analysis somewhat more difficult due to the fact that small-range attractive forces also arise due to the break-up of the double layer repulsion and subsequent effect of the attractive (van der Waals) force experienced by the tip in the proximity of the surface. The presence of increased attractive forces and decreased electrostatic repulsion on the nodules of charged surfaces compared to the uncharged can additionally result in increased adhesion. It is therefore possible to elucidate the fundamental surface forces that play a role in the nanoscale and molecular interactions at these polymer surfaces in liquid.

Individual force curves were fitted to DLVO models to assess the relative magnitude of the excess surface charges and potentials. In total eight force curves per sample were analysed, including fitting comparisons between force curves taken on the nodules and peripheries, for both uncharged and charged films. The significance of possible variation in the surface potentials between the samples was assessed by Student t-test at significance level of 0.05. The linear DLVO model comprising a single exponential function fitted reasonably well to the data (non-weighted $\chi^{2}, \mathrm{R}>0.96$ ) using the two parameter (surface potential and Debye length) model 
and the fitted curves were consistent with the theoretical Debye length of the $0.005 \mathrm{M} \mathrm{NaCl}(\mathrm{aq})$ electrolyte $(4.3 \mathrm{~nm})$. Figure 8 shows representative examples of the fitting for force curves taken from the nodule regions of the uncharged (Fig. 8A) and charged surfaces (Fig. 8B). Long range repulsion forces on the order of $10-100 \mathrm{pN}$ were present in all analysed curves and attributable to double layer electrostatics of a slightly negatively charged surface and negatively charged AFM tip (-200 mV). For example, the quantified surface potential was in the range of $-5 \mathrm{mV}$ to -50 $\mathrm{mV}$ for the films. Initial qualitative observations showed that the repulsive forces were weaker for the charged films compared to the uncharged films, as expected due to the application of a positive bias $(+200 \mathrm{mV})$ to these surfaces and quantitatively supported by the data fitting to the force curves. From the DLVO fitting, it was confirmed that the nodules of the charged film were significantly more positive than the nodules of the uncharged film $(p<.001$ at significance level of 0.05) but there was no significant difference in the estimated surface potentials of the peripheries of the uncharged and charged films ( $p=0.11$ at significance level of 0.05 ). When comparing differences across the polymer surfaces, the estimated surface potential of the nodules were slightly but not significantly more positive than that of the peripheries of the uncharged surfaces ( $p=0.086$ at significance level of 0.05 ). In contrast, the estimated surface potential of the nodules was significantly higher than that of the peripheries ( $p<.001$ at significance level of 0.05 ) for the charged films.

It is noted that the nonlinear DLVO model (eq.2, Fig. 8A), which is more appropriate for larger surface potentials, did not fit the data as well as the linear model and hence showed larger error in the calculated surface potential values. The nonlinear model also estimated shorter Debye lengths $(3.0 \mathrm{~nm})$ that were not consistent with expected theoretical values $(4.3 \mathrm{~nm})$. The weakness of the nonlinear model may be due to its greater sensitivity to error in defining the 
zero-distance (contact region) from the force curve recorded for soft materials such as the compliant PPy-HA films. Fitting the data to the nonlinear DLVO model (eq.2, Fig. 8A) systematically predicted $10-25 \mathrm{mV}$ more negative surface potential, both on the nodules and on the peripheries. Nonetheless, the general trends were identical to the linear model.

\section{Conclusions}

This work highlighted that the application of a relatively small potential bias of $+200 \mathrm{mV}$ to a PPy-HA film may have a large effect on the early attachment and spreading of hASCs. The majority of hASCs were uniformly adhered already at the three hour time-point on charged samples surfaces, however, cells seeded on non-charged PPy-HA surfaces were unevenly spread and many cells were not well adhered. The enhancement was significant with and without a preincubation period in cell culture medium containing plasma proteins. Pre-incubation alone had statistically equal significance in enhancing cell attachment. By using KPFM and AFM force mapping, it was possible to investigate the effects of charging on surface properties, including topography, modulus, surface potential and adhesion, with the intention of correlating these properties with the hASCs cell adhesion. As it turned out, charging did not have a significant effect on topography and only slightly increased the modulus but did significantly alter the surface potential and adhesion forces acting between the AFM tip and polymer. AFM force measurements with a negatively biased tip $(-200 \mathrm{mV})$ showed that prior to charging of the PPyHA films, short-range attractive forces and adhesion were restricted to the nodule peripheries but appeared on the nodules and uniformly across the film upon charging. The exact reason for such changes to occur only at the nodules is unclear, though these regions are known to be more highly doped and conductive. As expected, charging of the films increased the surface potential, as shown by both KPFM and DLVO analysis of the force curves, which had the effect of also 
decreasing the magnitude of the electrostatic repulsion between the tip and polymer. These changes in the interaction potential profile, namely the decreased repulsion and increased shortrange attraction and adhesion, give insight into the nature of the interaction forces that may play a role in promoting cell adhesion. Future work will concentrate on imaging the surface interactions of PPy-HA surface with AFM tip functionalized with plasma proteins and systematically adjusting salt concentration and $\mathrm{pH}$ to further investigate the double force electrostatics. 


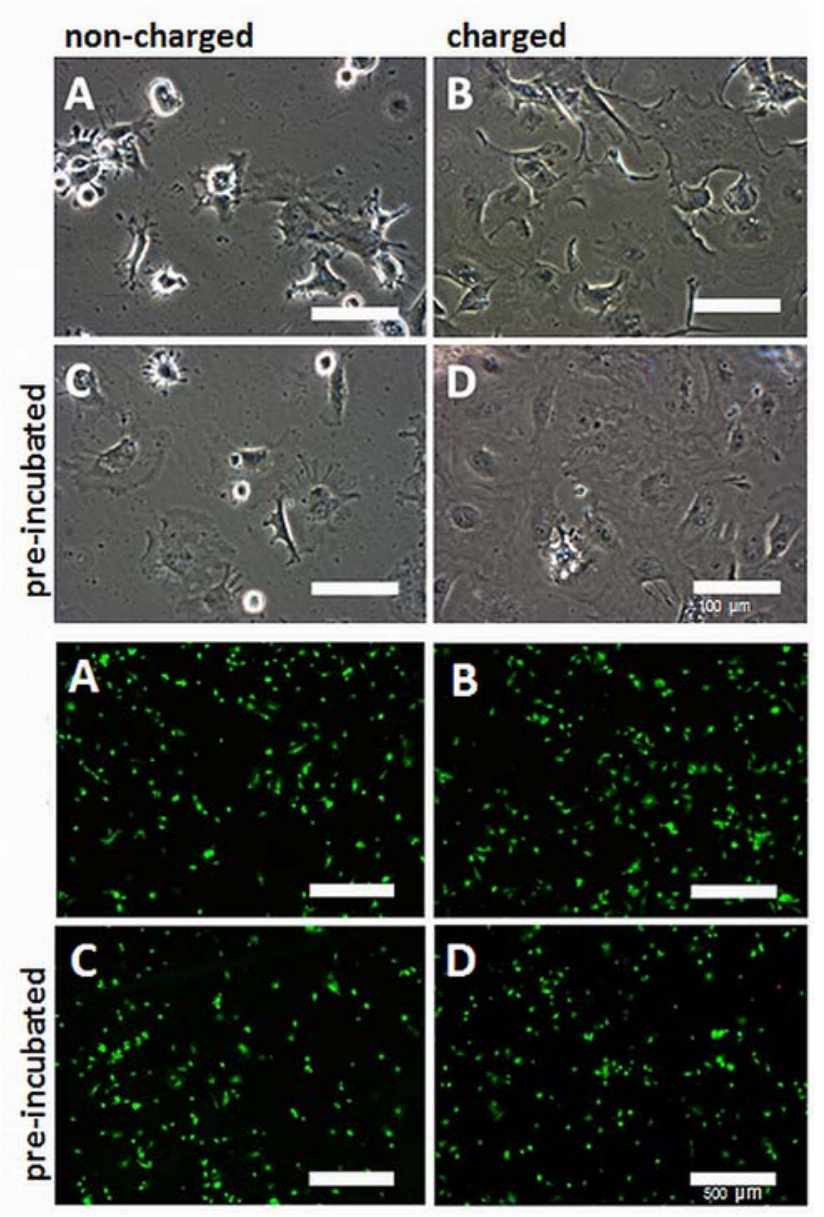

Figure 1. Top: Spreading of hASCs on non-preincubated (A and B), pre-incubated (C and D), and either charged (B and D) and uncharged (A and C) PPy-HA surface at 3h. Scale bars 100 $\mu \mathrm{m}$. Bottom: corresponding live/dead images, scale bar $500 \mu \mathrm{m}$. 


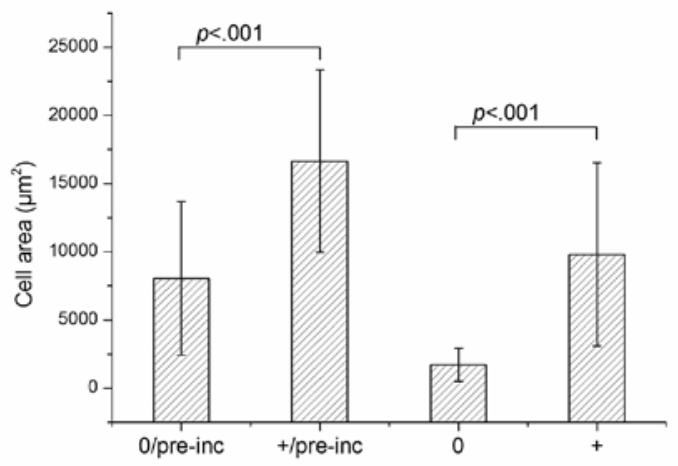

Figure 2. Average surface area of the hASCs at 3h, measured from three to five representative optical microscope images per group ( $n=26$ in each group). According to $t$-test and ANOVA the differences in cell areas were very significantly (at level of 0.01$)$ different $(p<.001)$ within the pre-incubated and the PBS rinsed groups but not between the (0/pre-inc) and (+) groups. 

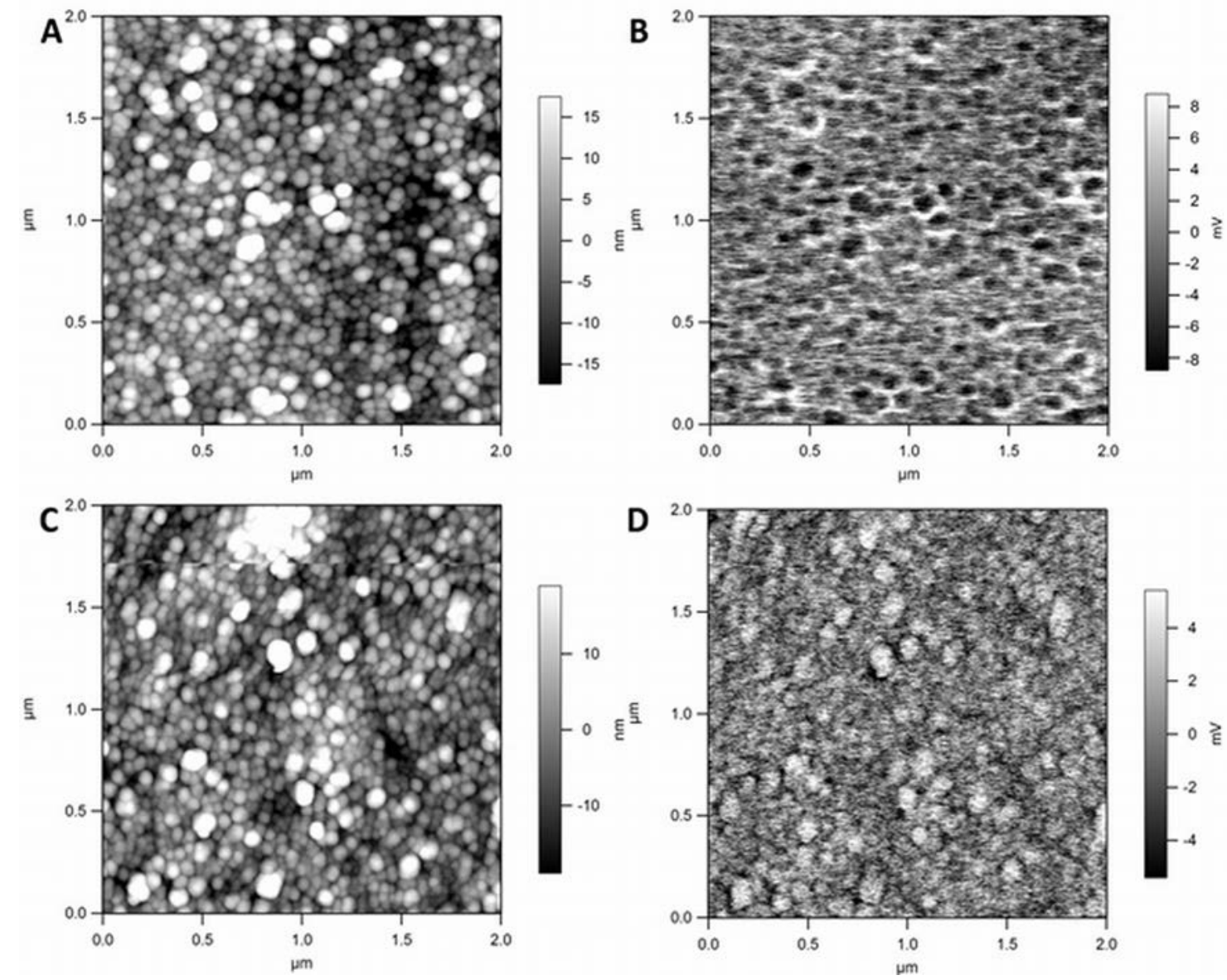

Figure 3. Kelvin Force Probe Microscopy. (A and B) Corresponding height (8.9 nm RMS) and surface potential images (4.38 mV RMS) for the uncharged PPy-HA film. (C and D) For the same film, corresponding height (9.4 nm RMS) and surface potential images (2.91 mV RMS) after charging (+200 mV). 

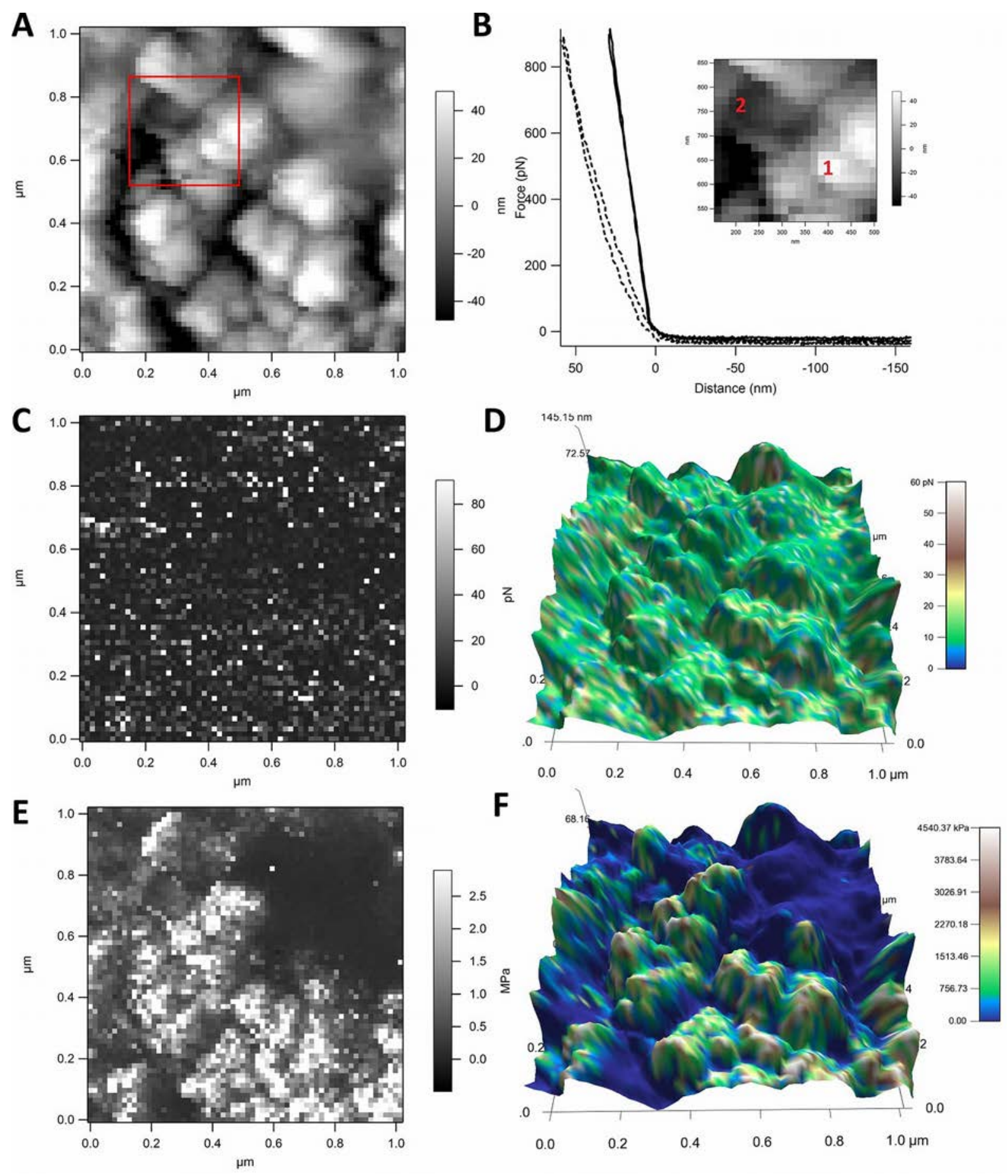

Figure 4. Uncharged Film - (A) Height image (23.7 nm RMS) obtained from the Force Map.

(B) Representative curves taken from the different regions (inset) of the film. Curves 1 and 2 are taken from nodules and peripheries, respectively. (C) Adhesion Map. (D) 3-D image of adhesion 
overlaid on topography. (E) Modulus Map (2.3 MPa RMS) (F) 3-D image of modulus overlaid on topography. Tip potential $0 \mathrm{mV}$. 

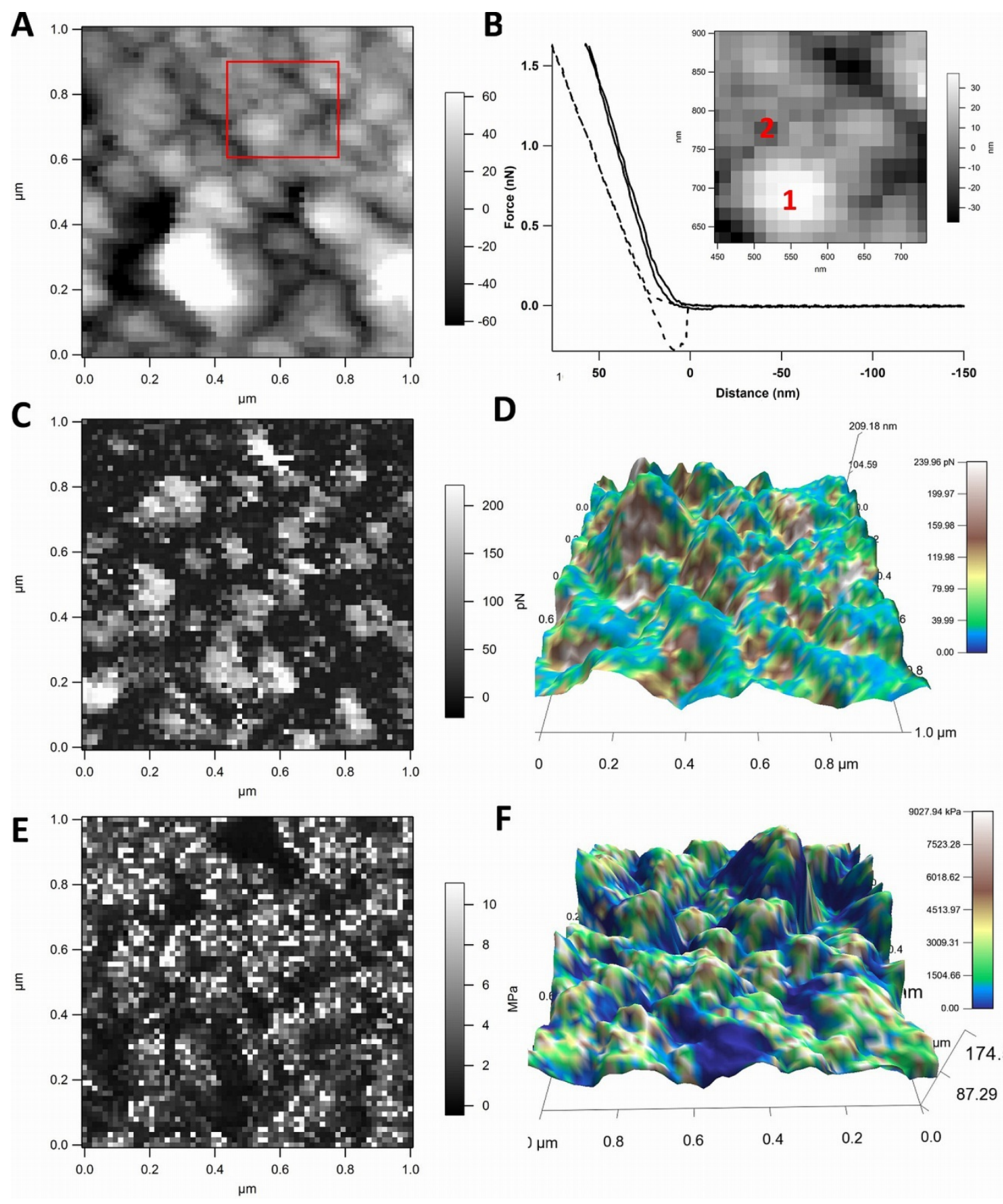

$\mathbf{F}$

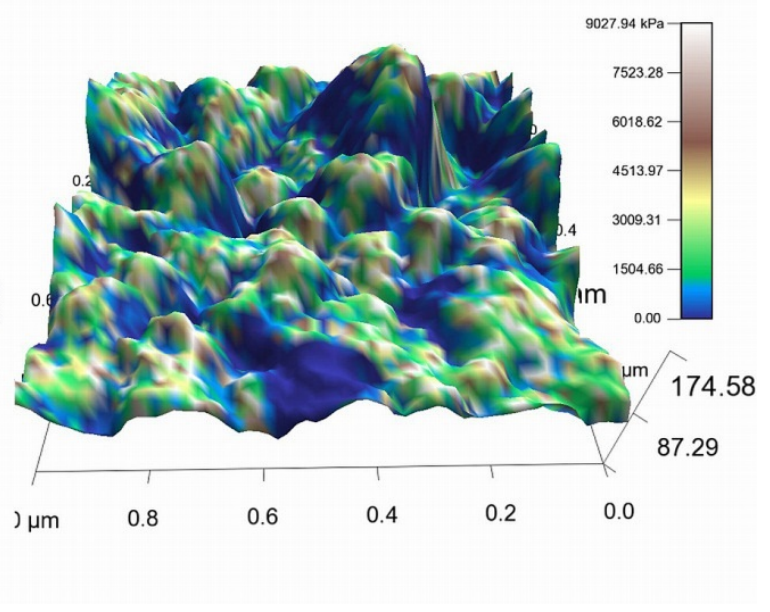

Figure 5. Charged Film (+200 mV) - (A) Height image (29.2 nm RMS) obtained from Force Map. (B) Representative curves taken from the different regions (inset) of the film. Curves 1 and 2 are taken from nodules and peripheries, respectively. (C) Adhesion Map (634 pN RMS) (D) 3- 
D image of adhesion overlaid on topography. (E) Modulus Map (5.3 MPa RMS). (F) 3-D image of modulus overlaid on topography. Tip potential $0 \mathrm{mV}$. 

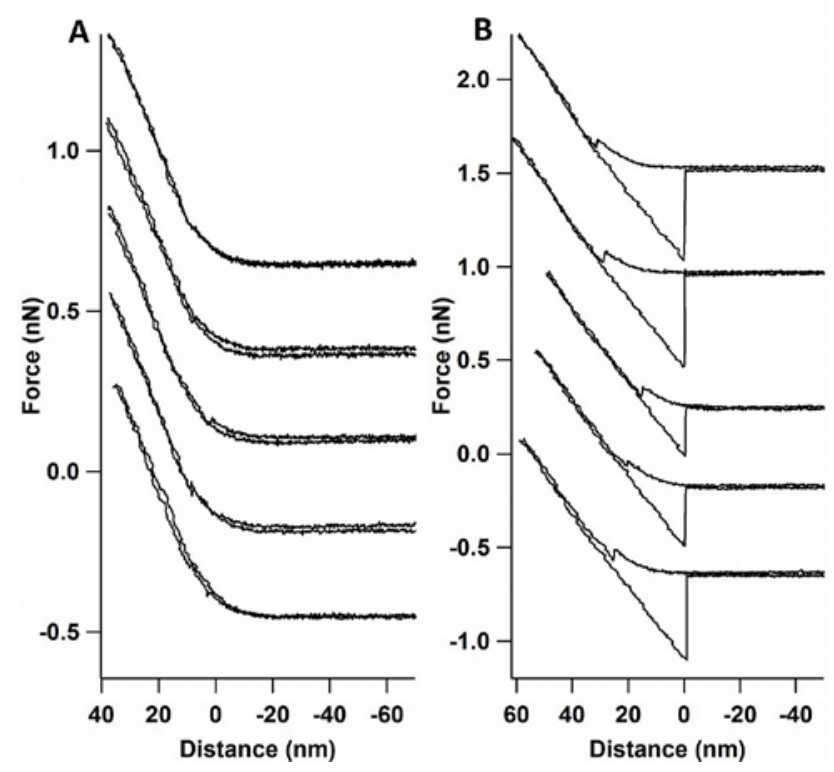

Figure 6. Force-Distance curves for an uncharged (0 mV) PPy-HA film. (A) the approach curve and the retracting curve on the nodules. (B) the approach curve and the retraction curve on the peripheries of the nodules. Tip potential $-200 \mathrm{mV}$ in $0.005 \mathrm{M} \mathrm{NaCl}(\mathrm{aq})$ electrolyte.
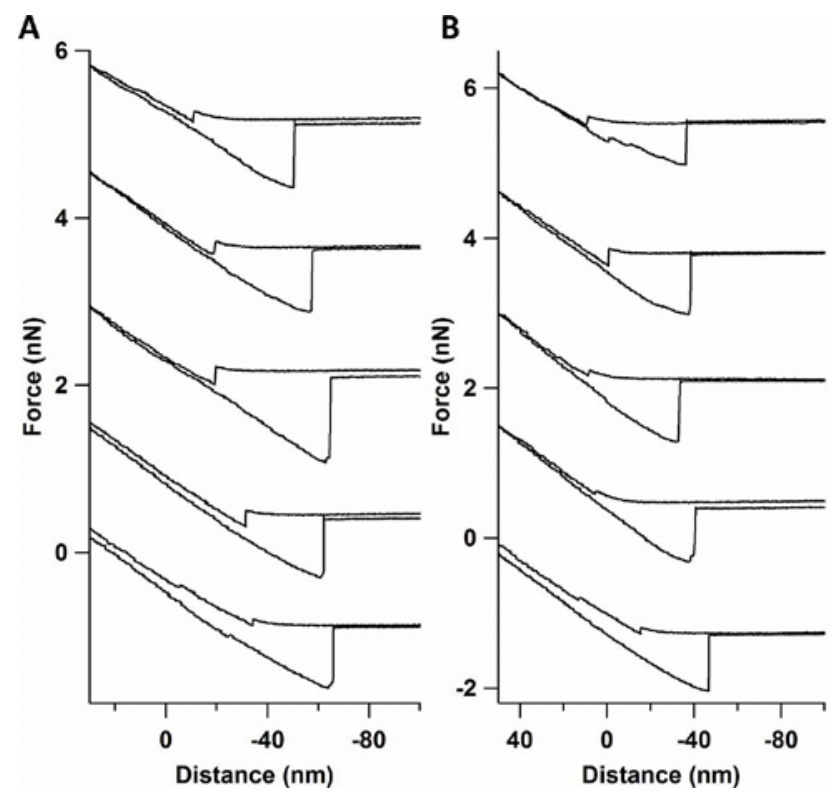

Figure 7. Force-Distance curves for a charged (+200 mV) PPyHA film. (A) the approach curve and the retracting curve on the nodules. (B) the approach curve and the retraction curve on the peripheries of the nodules. Tip potential $-200 \mathrm{mV}$ in $0.005 \mathrm{M} \mathrm{NaCl}(\mathrm{aq})$ electrolyte. 

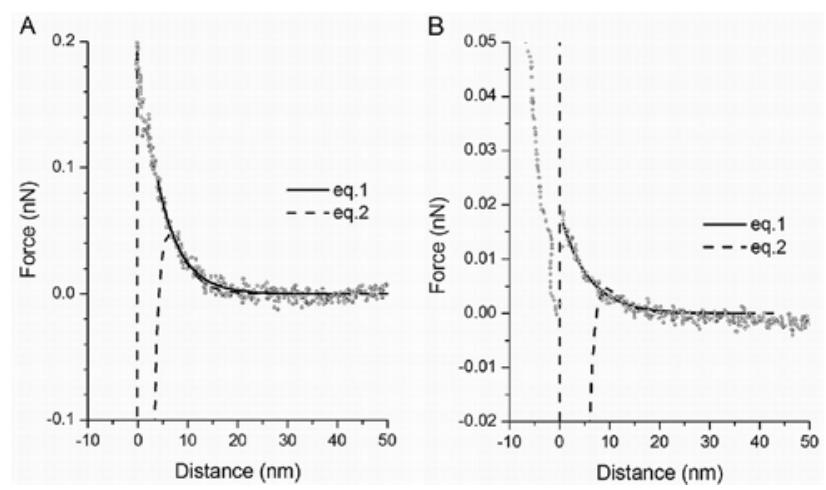

Figure 8. Typical approach curves and data fitting to simple exponential model according to Ohshima $^{20}$ (eq.1, solid line) and a nonlinear model according to Hull and Kitchener ${ }^{21}$ (eq.2, dashed line). (A) uncharged PPy-HA and (B) charged PPy-HA films. Tip potential -200 mV in 0.005M NaCl (aq) electrolyte. 
Table 1. PPyHA samples were categorized in 4 groups according to the pre-treatment and charging conditions.

\begin{tabular}{|l|l|l|}
\hline abbreviation & pre-treatment prior to ASC seeding and charging & $\begin{array}{l}\text { electrochemical } \\
\text { charging }\end{array}$ \\
\hline 0/pre-inc & pre-incubated in maintenance medium 48h & set to $0 \mathrm{mV}$ \\
\hline+ pre-inc & pre-incubated in maintenance medium $48 \mathrm{~h}$ & oxidized at $+200 \mathrm{mV}$ \\
\hline 0 & rinsing with PBS & set to $0 \mathrm{mV}$ \\
\hline+ & rinsing with PBS & oxidized at $+200 \mathrm{mV}$ \\
\hline
\end{tabular}


AUTHOR INFORMATION

* Corresponding author: Jani Pelto

Tel. +358 207223571, Fax. +358 207223498, Email. Jani.Pelto@vtt.fi

\section{Author Contributions}

The manuscript was written through contributions of all authors. All authors have given approval to the final version of the manuscript.

\section{ACKNOWLEDGMENT}

The support of this work by The Finnish Funding Agency for Technology and innovation (TEKES) is gratefully acknowledged. This work has been supported by the Australian Research Council under the Australian Research Fellowship and DP110104359 (Dr Michael Higgins) and ARC Federation Fellowship of Prof. Gordon Wallace. We also greatly acknowledge the Australian National Fabrication Facility (ANFF) for providing Atomic Force Microscopy instrumentation. The authors thank Miss. Anne Rajala and Miss. Leena Jaatinen for the preparation of the samples.

\section{ABBREVIATIONS}

DMEM, Dulbecco’s Modified Eagle Medium; DPBS, Dulbecco’s Phosphate-Buffered Saline; FACS, fluorescence-activated cell sorting; CD, cluster of differentiation (e.g. CD10-PECy7, CD10 transmembrane protein, fluorescence labeled with R-phycoerythrin cyanine-7 conjugate). 


\section{REFERENCES}

(1) Collier, J.; Camp, J.; Hudson, T.; Schmidt, C. Synthesis and characterization of polypyrrole-hyaluronic acid composite biomaterials for tissue engineering applications. $J$. Biomed. Mater. Res. 2000, 50, 574-584.

(2) Zhang, X.; Bai, R. Surface electric properties of polypyrrole in aqueous solutions. Langmuir 2003, 19, 10703-09.

(3) Pelto, J.; Haimi, S.; Puukilainen, E.; Whitten, P,; Spinks, G.; Bahrami-Samani, M.; et al. Electroactivity and biocompatibility of polypyrrole hyaluronic acid multi-walled carbon nanotube composite. J. Biomed. Mater. Res. A 2010, 93, 1056-67.

(4) Pei, Q.; Inganäs, O. Electrochemical applications of the bending beam method. Mass transport and volume changes in polypyrrole during redox. J. Phys. Chem. 1992, 96, 10507-14.

(5) Serra Moreno, J.; Panero, S.; Materazzi, S.; Martinelli, A.; Sabbieti, M.; Agas, D.; et al. Polypyrrole-polysaccharide thin films characteristics: Electrosynthesis and biological properties. J. Biomed. Mater. Res. A 2009, 88, 832-40.

(6) Lee, J.; Schmidt, C. Pyrrole-hyaluronic acid conjugates for decreasing cell binding to metals and conducting polymers. Acta Biomater. 2010, 6, 4396-4404.

(7) Gelmi, A.; Higgins, M.; Wallace, G. Physical surface and electromechanical properties of doped polypyrrole biomaterials. Biomaterials 2010, 31, 1974-83. 
(8) Gilmore, K.; Kita, M.; Han, Y.; Gelmi, A.; Higgins, M.; Moulton, S.E.; et al. Skeletal muscle cell proliferation and differentiation on polypyrrole substrates doped with extracellular matrix components. Biomaterials 2009, 30, 5292-5304.

(9) Dubiel, E.; Martin, Y.; Vermette, P. Bridging the Gap between physicochemistry and interpretation prevalent in cell-surface interactions. Chem. Rev. 2011, 111, 2900-36.

(10) Lubarsky, G.; Browne, M.; Mitchell, S.; Davidson, M.; Bradley, R. The influence of electrostatic forces on protein adsorption. Colloids Surf. B: Biointerfaces 2005, 44, 56-63.

(11) Wang, J.; Bard, A. Direct atomic force microscopic determination of surface charge at the gold/electrolyte interface-the inadequacy of classical GCS theory in describing the double-layer charge distribution. J. Phys. Chem. B 2001, 105, 5217-22.

(12) Discher, D.; Janmey, P.; Wang, Y. Tissue cells feel and respond to the stiffness of their substrate. Science 2005, 310, 1139-43.

(13) De Santis, G.; Lennon, A.; Boschetti, F.; Verhegghe, B.; Verdonck, P.; Prendergast, P. How can cells sense the elasticity of a substrate? An analysis using a cell tensegrity model. Eur. Cells Mater. 2011, 22, 202-13.

(14) Gimble, J.; Guilak, F. Adipose-derived adult stem cells: Isolation, characterization, and differentiation potential. Cytotherapy 2003, 5, 362-69.

(15) Gronthos, S.;Franklin, D.; Leddy, H.; Robey, P.; Storms, R.; Gimble, J. Surface protein characterization of human adipose tissue-derived stromal cells. J. Cell Physiol. 2001, 189, 54-63. 
(16) Lindroos, B.; Boucher, S,; Chase, L.; Kuokkanen, H.; Huhtala, H.; Haataja, R.; et al. Serum-free, xeno-free culture media maintain the proliferation rate and multipotentiality of adipose stem cells in vitro. Cytotherapy 2009, 11, 958-72.

(17) Haimi, S.; Pirhonen, E.; Moimas, L.; Lindroos, B.; Huhtala, H.; Räty, S.; et al. Calcium phosphate surface treatment of bioactive glass causes a delay in early osteogenic differentiation of adipose stem cells. J. Biomed. Mater. Res. A 2009, 91, 540-7.

(18) Haimi, S.; Suuriniemi, N.; Haaparanta, A-M.; Ellä, V.; Lindroos, B.; Huhtala, H.; et al. Growth and osteogenic differentiation of adipose stem cells on PLA/bioactive glass and PLA/ $\beta$ TCP scaffolds. Tissue Eng. Pt A 2009;15:1473-80.

(19) Sader, J.; Chon, J.; Mulvaney, P. Calibration of rectangular atomic force microscope cantilevers. Rev. Sci. Instrum. 1999, 70, 3967-69.

(20) Ohshima, H. Electrostatic interaction between a sphere and a planar surface: Generalization of point-charge/surface image interaction to particle/surface image interaction. $J$. Colloid Interface Sci. 1998, 198, 42-52.

(21) Hull, M.; Kitchener, J. Interaction of spherical colloidal particles with planar surfaces. Trans. Faraday Soc. 1969, 65, 3093-3104.

(22) Higgins, M.; Molino, P.; Yue, Z.; Wallace, G. Organic conducting polymer-protein interactions. Chem. Mater. 2012, 24, 828-39.

(23) Barisci, J.; Stella, R.; Spinks, G.; Wallace, G. Characterization of the topography and surface potential of electrodeposited conducting polymer films using atomic force and electric force microscopies. Electrochim. Acta 2000, 46, 519-31. 
(24) O'Neil, K.; Shaw, B.; Semenikhin, O. On the origin of mesoscopic inhomogeneity of conducting polymers. J. Phys. Chem. B 2007, 111, 9253-69.

(25) Gelmi, A.; Higgins, M.; Wallace, G. Attractive and repulsive interactions originating from lateral nanometer variations in surface charge/energy of hyaluronic acid and chondroitin sulfate doped polypyrrole observed using atomic force microscopy. J. Phys. Chem. B 2012, 116, 13498505.

(26) Bay, L.; Jacobsen, T.; Skaarup, S.; West, K. Mechanism of actuation in conducting polymers: Osmotic expansion. J. Phys. Chem. B 2001, 105, 8492-97. 